\title{
Artéria radial na ampliação do uso de enxertos arteriais para revascularização do miocárdio: considerações anatômicas e tática cirúrgica
}

\author{
Luís Alberto DALLAN*, Sérgio Almeida de OLIVEIRA*, Fábio B. JATENE*, Ricardo \\ CORSO*, José Carlos R. IGLESIAS*, Nadir PRATES ${ }^{\star \star *}$, Januário M. SOUZA**, Geraldo \\ VERGINELLI*, Adib D. JATENE*
}

RBCCV $44205-293$

\begin{abstract}
Dallan L A, Oliveira S A, Jatene F B, Corso R, Iglésias J C, Prates N, Souza J M, Verginelli G, Jatene A D Artéria radial na ampliação do uso de enxertos arteriais para revascularização do miocárdio: consideraçōes anatômicas e tática cirúrgica. Rev Bras Cir Cardiovasc 1996; 11 (2): 75-81.
\end{abstract}

\begin{abstract}
RESUMO: A artéria radial (AR) foi estudada sob o ponto de vista anatômico, histológico, imunohistoquímico e ultramicroscópico. Paralelamente, foram analisados os resultados de seu emprego na revascularização do miocárdio de 269 pacientes. No total, 319 artérias coronárias foram revascularizadas pela AR. Houve predomínio do sexo masculino $(80,7 \%)$ e da raça branca $(93,7 \%)$. A artéria torácica interna esquerda (ATle) foi empregada em 246 pacientes. Outros 17 já haviam sido previamente revascularizados com a ATle, perfazendo um total de $97,8 \%$. A artéria torácica interna direita (ATId) foi empregada em 59 $(21,9 \%)$ pacientes, a artéria gastroepiplóica direita (AGEd) em $17(6,3 \%)$ pacientes e em $161(59,8 \%)$ foram também realizadas 1 a 4 pontes de veia safena. A média de enxertos por paciente foi de 3,4 . Não houve complicaçōes isquêmicas ou infecciosas no membro em que a AR foi retirada. Os estudos imuno-histoquímicos e ultramicroscópicos permitiram observar o grande espessamento da membrana limitante elástica interna da AR e o predomínio de fibras colágenas sobre as elásticas na camada média. As principais complicaçōes pósoperatórias foram a fibrilação atrial em $21(7,8 \%)$ pacientes, insuficiência respiratória em $21(7,8 \%)$, alterações no ECG e/ou elevação de CKMB em $12(4,5 \%)$ pacientes. O balão intra-aórtico foi utilizado em $6(2,2 \%)$ pacientes. O cateterismo no pós-operatório precoce foi realizado em 21 pacientes. Em 1 paciente houve dissecção sem oclusão do óstio da AR pelo cateter, $1(4,7 \%)$ apresentou sinais de espasmo da AR; nos demais a AR encontrava-se pérvia e sem lesōes. Houve $6(2,2 \%)$ óbitos hospitalares, sendo 3 por acidente vascular cerebral, 2 por baixo débito cardíaco e 1 paciente teve morte súbita. Nos últimos meses temos visado à revascularização miocárdica com o emprego máximo de enxertos arteriais. Como tática especial usamos a AR para anastomoses seqüenciais com as artérias de parede inferior e lateral do ventrículo esquerdo. A anastomose do coto proximal é realizada na ATle que, por sua vez, é habitualmente anastomosada ao ramo interventricular anterior (RIA). As artérias AGEd e ATId in situ ou, como enxerto livre, têm complementado o procedimento. Dentre 64 pacientes operados nos últimos 3 meses com a utilizaçāo da AR, $62(96,9 \%)$ receberam pelo menos 2 enxertos arteriais, $27(42,2 \%)$ receberam pelo menos 3 enxertos arteriais e $8(12,5 \%)$ pacientes receberam 4 ou 5 enxertos arteriais. Em $29(45,3 \%)$ deles a AR foi anastomosada à ATle ou ATId. Acreditamos que essa técnica reduza a excessiva pressāo conseqūente à anastomose direta da AR na aorta e que o maior fluxo decorrente das anastomoses seqũenciais auxilie na manutenção da perviabilidade da AR. A despeito de considerarmos o enxerto pediculado da ATle o principal método de revascularização do miocárdio, os resultados preliminares indicam que a AR constitui uma excelente via complementar na busca da revascularização completa do miocárdio com enxertos arteriais.
\end{abstract}

DESCRITORES: Arteria radial, transplante. Revascularizaçāo do miocárdio, métodos. Artérias torácicas, cirurgia. Aorta, cirurgia. Veia safena, cirurgia. 
Dallan L A, Oliveira S A, Jatene F B, Corso R, Iglésias J C, Prates N, Souza J M, Verginelli G, Jatene A D - Artéria radial na ampliação do uso de enxertos arteriais para revascularização do miocárdio: consideraçōes anatômicas e tática cirúrgica. Rev Bras Cir Cardiovasc 1996; 11 (2): 75-81.

\section{INTRODUÇÃO}

O emprego de enxertos arteriais na revascularização do miocárdio vem sendo ampliado. Já têm sido estabelecidas as vantagens da artéria torácica interna (ATI) sobre os enxertos com veia safena, proporcionando melhor longevidade e qualidade de vida aos pacientes (1-3). Entretanto, existem situaçōes em que apenas as 2 ATls não são suficientes para a completa revascularização miocárdica, exigindo enxertos alternativos. Nesses casos estão incluídos pacientes com múltiplas obstruções arteriais coronárias, pacientes com contra-indicação para o uso de ambas ATIs, especialmente pacientes idosos insulino-dependentes, pacientes safenectomisados ou com insuficiência vascular periférica, ou nas reoperações em que esses enxertos já tenham sido empregados ${ }^{(4)}$. A artéria gastroepiplóica direita (AGEd), a artéria epigástrica inferior $(A E I)$ e a artéria radial (AR) são enxertos arteriais propostos recentemente na revascularização do miocárdio, alguns, inclusive, com controvérsias de sua eficácia inicial $(5-13)$.

O objetivo deste trabalho foi trazer subsidios sobre as características anatômicas e ultramicroscópicas da AR e destacar diversas variaçōes técnicas na revascularização do miocárdio com esse tipo de enxerto.

\section{CASUÍSTICA E MÉTODOS}

Entre maio/94 e dezembro/95, estudamos 269 pacientes que receberam 1 ou mais enxertos de AR. No total, 319 artérias coronárias foram revascularizadas pela AR. Houve predomínio do sexo masculino $(80,7 \%)$ e da raça branca $(93,7 \%)$. A faixa etária variou de 30 a 81 anos $(M=58,1$ anos). $A$ ATle foi empregada em 246 pacientes. Em outros 17 pacientes já revascularizados previamente preservou-se a ATle (que se encontrava funcionante), perfazendo $263 / 269(97,8 \%)$ pacientes com concomitante enxerto da ATle nesse grupo. A ATId foi empregada em $59(21,9 \%)$ pacientes, a AGEd em $17(6,3 \%)$ (Figura 1) e em $161(59,8 \%)$ foram também realizadas 1 a 4 pontes de veia safena. A média de enxertos por paciente foi de 3,4. Trinta e um $(11,5 \%)$ pacientes dessa casuística tinham revascularização prévia, sendo 25 na primeira reoperação, 5 na segunda e 1 na terceira. Concomitantemente, foi realizada troca de valva aórtica em 2 pacientes, plástica de valva mitral em 2 pacientes, aneurismectomia de ventrículo esquerdo em 6 pacientes e endarterectomia de artéria carótida em 1. A anastomose da AR foi feita diretamente na aorta em $214(79,5 \%)$ pacientes, sobre veia safena em $26(9,7 \%)$, com a ATle em $24(8,9 \%)$ (Figura 2) e com ATId em 5 (1,9\%). Em $36(13,4 \%)$ pacientes

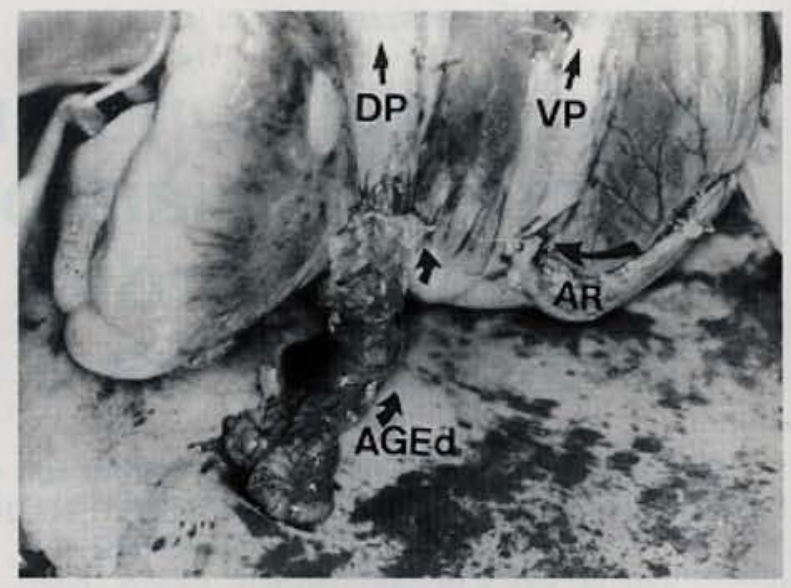

Fig. 1 - Enxerto de art. gastroepiplóica D (AGEd) para ramo interventricular posterior (DP) da CD. Anastomose terminal da art. radial (AR) para ramo ventricular posterior (VP) da artéria circunflexa. $(\boldsymbol{-})$

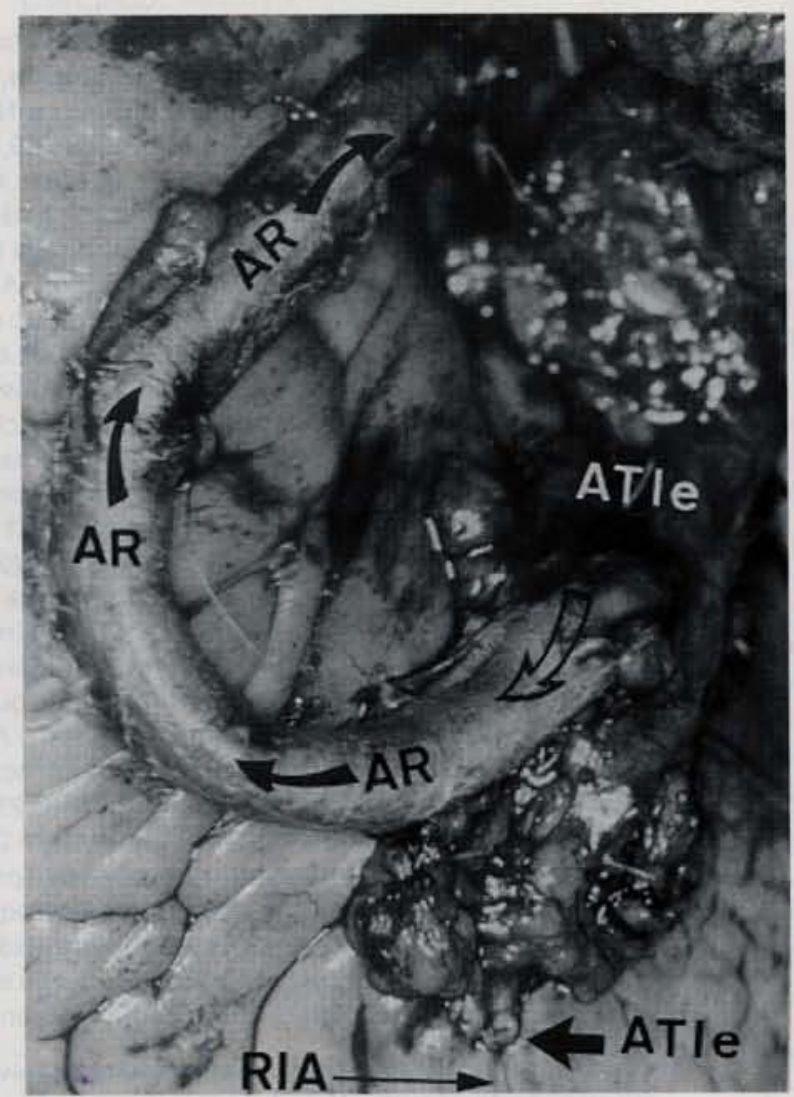

Fig. 2 - Anastomose da art. torácica interna $E$ (ATle) para ramo interventricular anterior (RIA). $(\rightarrow$ ) Anastomose da art. radial (AR) na ATle, em seu fluxo natural constituindo " $Y$ " artificial (i) .

a AR foi usada em seqüência para 2 artérias coronárias e em $7(2,6 \%)$ para 3 artérias coronárias (Figuras 3 e 4 ). 
Dallan L A, Oliveira S A, Jatene F B, Corso R, Iglésias J C, Prates N, Souza J M, Verginelli G, Jatene A D - Artéria radial na ampliação do uso de enxertos arteriais para revascularização do miocárdio: consideraçōes anatômicas e tática cirúrgica. Rev Bras Cir Cardiovasc 1996; $11(2): 75-81$.

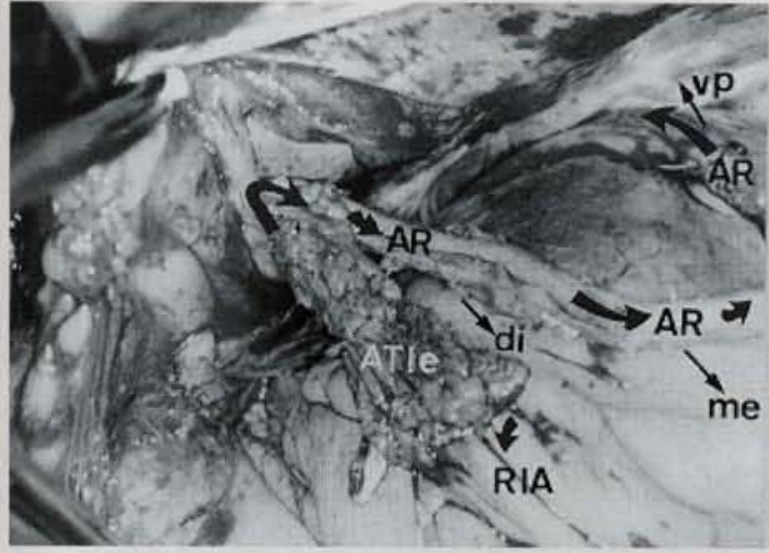

Fig. 3- Anastomose da artéria torácica interna $E$ (ATle) para o ramo interventricular anterior (RIA) $(-)$. Anastomose da arteria radial (AR) na ATle e sequencial para os ramos diagonal (di), marginal $E$ (me) e ventricular posterior (VP) da coronária esquerda.

\section{Técnica de Preparo da Artéria Radial}

O adequado conhecimento da anatomia do antebraço é necessário para a retirada da AR com segurança. $O$ estudo através do eco-Doppler e/ou manobra de Allen deve mostrar o fluxo adequado das artérias radial e ulnar, assim como a existência dos arcos palmares superficial e profundo. A incisão da pele e subcutâneo é unica, iniciando-se poucos centímetros acima do punho, até atingir as proximidades da prega do cotovelo. Nesse local deve-se incisar e afastar lateralmente o músculō bráquioradial, evitando lesar estruturas noḅres, como a artéria braquial, a artéria ulnar e o nervo mediano (15). No terço médio do antebraço, deve-se ter cuidado em preservar o ramo superficial do nervo radial, responsável pela sensibilidade cutânea de porções do antebraço, polegar e dorso da mão (15). O ramo superficial do nervo radial está intimamente relacionado à artéria, e sua dissecção grosseira, ou o afastamento lateral excessivo do músculo bráquioradial, pode lesá-lo, causando parestesia no polegar. Na porção distal do antebraço, a AR é bastante superficial, sendo recoberta apenas pela pele, tecido conjuntivo e fáscia superficial. Entretanto, os ramos perfurantes da $A R$ nesse local são mais curtos, finos e delicados que nos outros dois terços do antebraço, exigindo ligadura mais cuidadosa. A AR foi retirada preferencialmente do membro superior com menor dominância. Nas reoperações, optou-se pela artéria não submetida à cateterização contínua na operação anterior. A AR foi retirada em conjunto com suas duas veias satélites. Nas anastomoses da AR com as artérias coronárias com a aorta ou com as artérias torácicas internas, utilizou-se sutura contínua com Polipropileno 7,0 ou 8,0 .

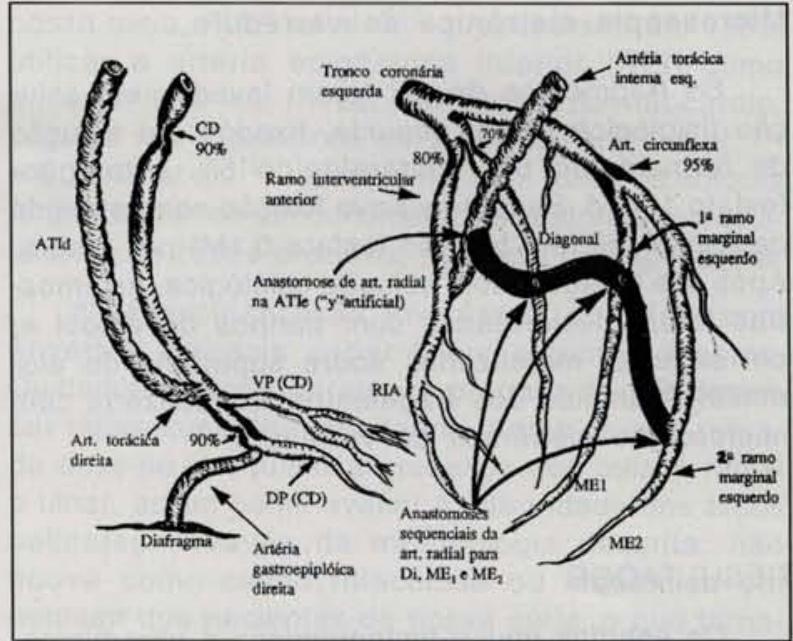

Fig. 4 - Aspecto esquemático da revascularização completa do miocárdio com enxertos arteriais, usando ATle, ATId, AR e AGEd.

Todos os pacientes receberam nifedipina no intra e no pós-operatório, quando se associou o AAS.

\section{Estudos Anatômicos}

Estudos da estrutura da AR incluíram imuno-histoquimica para elastina, microscopia eletrônica de varredura e diferenciação histológica entre fibras colágenas e fibras elásticas por colorações específicas.

\section{Histologia}

Fragmentos da AR foram incluidos em blocos de parafina com $1 \mathrm{~cm} 2$ e cortados em unidades seriadas de 10 micrômetros. Foram realizadas colorações com hematoxilina-eosina, Weighert, picrus sirius e tricrômio de Masson. O estudo microscópico destacou a arquitetura do vaso e a distribuição do tecido conectivo e fibras elásticas.

\section{Imuno-histoquímica}

Fragmentos da AR foram fixados em paraformaldeído $4 \%$ em solução tampão-fosfato $0,1 \mathrm{M}$. Após $2 \mathrm{~h}$ a concentração da solução fixadora foi elevada para $8 \%$. Durante a inclusão em parafina, a temperatura foi mantida entre $50^{\circ} \mathrm{C}$ e $60^{\circ} \mathrm{C}$. As amostras foram, a seguir, incubadas por $2 \mathrm{~h}$ em câmara úmida a $37^{\circ} \mathrm{C}$ com a primeira solução de anticorpos (anticolágeno, antifibrina e antielastina). Após nova lavagem, as amostras foram reincubadas por $1 \mathrm{~h}$ com anticorpos marcados com fluoresceína. As lâminas foram, então, examinadas em microscópio fluorescente. 
Dallan L A, Oliveira S A, Jatene F B, Corso R, Iglésias J C, Prates N, Souza J M, Verginelli G, Jatene A D - Artéria radial na ampliaçāo do uso de enxertos arteriais para revascularização do miocárdio: consideraçōes anatômicas e tática cirúrgica. Rev Bras Cir Cardiovasc 1996; 11 (2): 75-81.

\section{Microscopia eletrônica de varredura}

Os fragmentos de AR foram lavados em solução fisiológica e, em seguida, fixados em solução de formaldeído $6 \%$, glutaraldeído $6 \%$ e tampãofosfato $10,1 \mathrm{M}$. Seguiu-se nova fixação com tetróxido de ósmio $1 \%$ (em tampão fosfato $0,1 \mathrm{M}$ ) por 1 hora. Após sua lavagem com solução fisiológica, as amostras foram desidratadas com banhos de álcool e, em seguida, metalizadas sobre superfície de alumínio. A análise dos fragmentos foi realizada com microscópio eletrônico de varredura.

\section{RESULTADOS}

Os estudos imuno-histoquímicos e ultramicroscópicos permitiram observar o grande espessamento da limitante elástica interna da $\mathrm{AR}$ e o predomínio das fibras colágenas sobre as elásticas em sua camada média. A microscopia eletrônica de varredura demonstrou que o endotélio da AR é formado por camada preenchida por estruturas circulares com inúmeras dobras, o que lhe confere aspecto de "tronco de árvore" (Figura 5). a camada média apresenta fibras musculares circundadas por estruturas lineares, correspondentes a fibras colágenas (Figura 6). É possível, também, observarem-se, em determinados pontos dessa camada, fibras elásticas semelhantes a "cabelo de anjo", entremeadas às fibras colágenas (Figura 7). A camada adventícia é formada por uma fina membrana, com inúmeras projeçōes em sua porção mais superficial, mimetizando "dedos de luva".

Não houve complicações isquêmicas ou infecciosas no membro em que a $A R$ foi retirada. Dezesseis $(5,9 \%)$ pacientes relataram discreta parestesia transitória no polegar correspondente. $O$ período médio de internação pós-operatória foi de 8,4

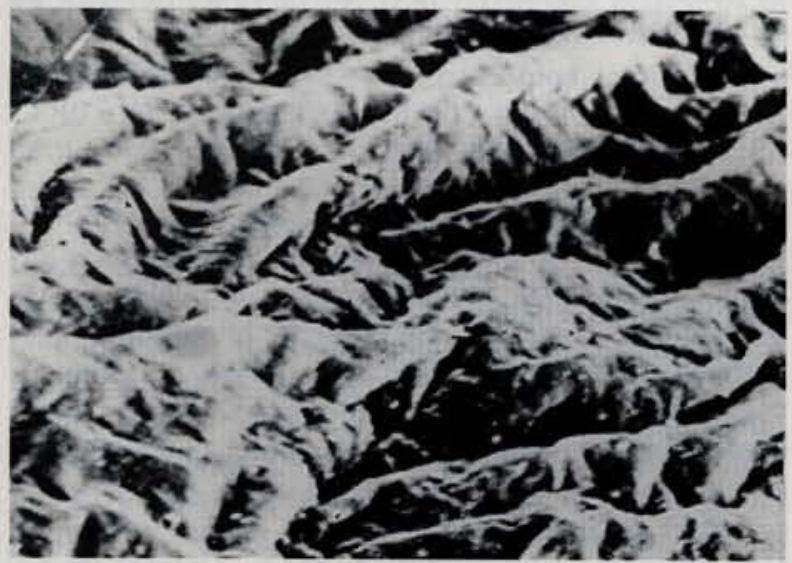

Fig. 5 - Microscopia eletrônica de varredura: endotélio da AR com aspecto de "tronco de árvore" ( $\mathrm{x} 2000$ )

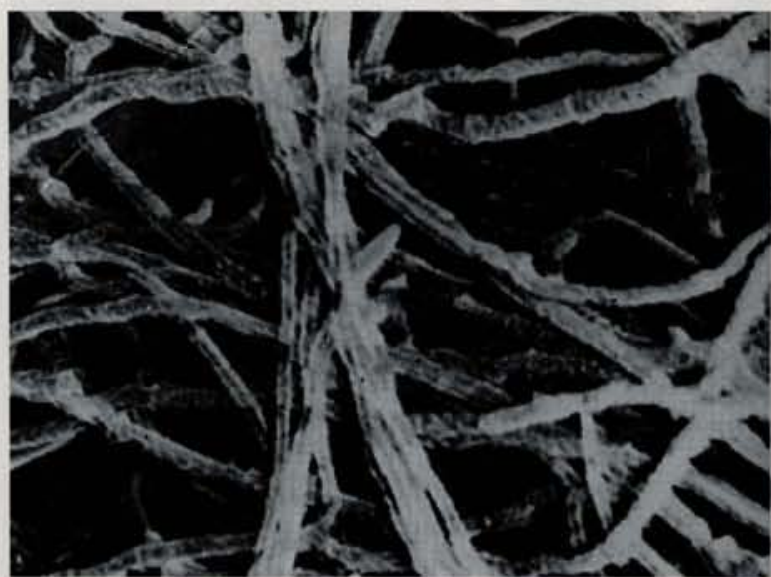

Fig. 6- Aspecto ultramicroscópico das fibras colágenas da camada média da $A R$, mais concentradas próximo à camada adventicia $(\times 5000)$.

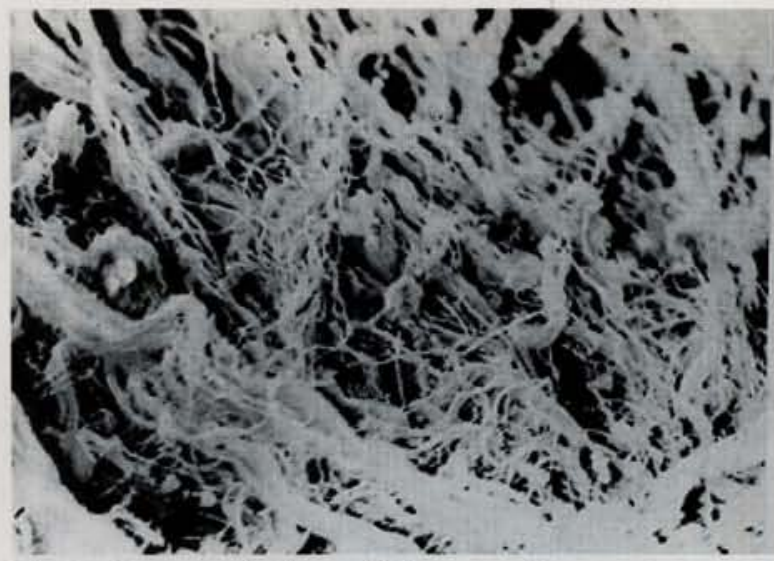

Fig. 7 - Presença de fibras elásticas semelhantes a "cabelos de anjo"em meio às fibras colágenas da camada média da AR. Microscopia eletrônica de varredura (x 3000).

dias. Como principais complicações destacaram-se: fibrilação atrial $(21-7,8 \%)$, insuficiência respiratória (21-7,8\%), alteração de ECG e/ou elevação de CKMB $(12-4,5 \%)$. O balão intra-aórtico foi utilizado em 6 $(2,2 \%)$ pacientes. o cateterismo no pós-operatório precoce foi realizado em 21 pacientes. Em 1 houve dissecção sem oclusão do óstio da AR pelo cateter; $1(4,7 \%)$ paciente apresentou sinais de espasmo na AR; nos demais a AR encontrava-se pérvia e sem lesões (Figuras 8 e 9 ). Houve $6(2,2 \%)$ óbitos hospitalares, sendo 3 por acidente vascular cerebral, 2 por baixo débito cardíaco e 1 paciente teve morte súbita na enfermaria.

Nos últimos 3 meses, dentre 64 pacientes operados com a utilização da $A R, 62(96,9 \%)$ receberam pelo menos 2 enxertos arteriais, $27(42,2 \%)$ receberam pelo menos 3 enxertos arteriais e 8 (12,5\%) pacientes receberam 4 ou 5 enxertos arteriais. 
Dallan L A, Oliveira S A, Jatene F B, Corso R, Iglésias J C, Prates N, Souza J M, Verginelli G, Jatene A D - Artéria radial na ampliaçāo do uso de enxertos arteriais para revascularizaçăo do miocárdio: considerações anatômicas e tática cirúrgica. Rev Bras Cir Cardiovasc 1996; 11 (2): 75-81.

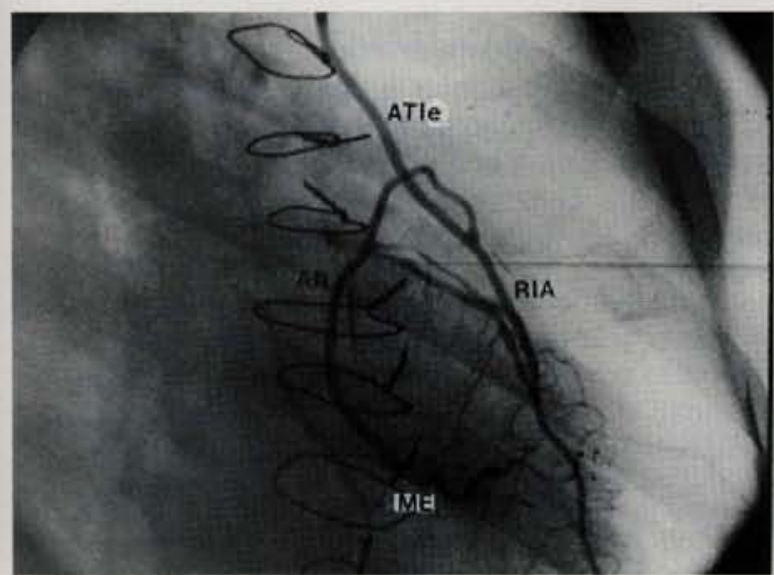

Fig. 8 - Estudo angiográfico pós-operatório. O contraste injetado na ATle permite visibilizar enchimento do RIA, artéria radial (AR) saindo em " $Y$ "da ATle e ramos marginais da artéria circunflexa (ME).

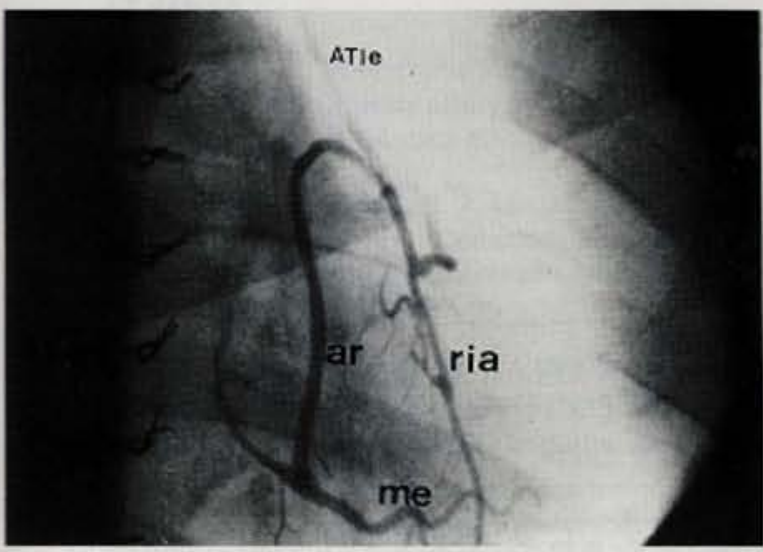

Fig. 9 - Estudo angiográfico pós-operatório mestrando a contrastação do ramo interatrial (ria), artéria radial (ar) e artéria marginal $E$ (me) a partir da art. torácica interna E (ATle).

\section{COMENTÁRIOS}

A anastomose da ATle ramo interventricular anterior (RIA) tem a sua eficiência demonstrada no decorrer dos últimos anos; com grande superioridade em relação aos enxertos com a veia safena (14). Bons resultados tardios também vêm sendo observados com o emprego de ambas ATIs $(15,16)$. Essas evidências estimularam a busca de outros enxertos visando à revascularização completa do miocárdio apenas com condutos arteriais. SUMA et al. (12) revelaram a viabilidade do emprego da AGEd in situ ou como enxerto livre, especialmente na revascularização de ramos da artéria coronária direita. ACAR et al. (5) retomaram o uso das artérias radiais, proposto e abandonado por CARPENTIER et al. (7) na década de 70 , demonstrando ser possivel contornar com novas técnicas e drogas o espasmo arterial que comprometia seu perfeito funcionamento. Em nosso meio, PUIG et al. (10) propuseram, em 1990, utilizar a artéria epigástrica inferior ( $A E I)$ como enxerto livre na revascularização do miocárdio, seguido nos 2 próximos anos por BARNER et al. (1) e BUCHE et al. (17). Entretanto, nem todos os autores foram unânimes em considerar satisfatórios os resultados com esses enxertos, no início da experiência.

A revascularização miocárdica completa com enxertos arteriais esbarra em algumas arestas. Cuidados básicos durante o preparo da AR devem ser rigorosamente respeitados. Antes de sua retirada deve-se pesquisar a presença dos pulsos radial e ulnar, assim como avaliar a integridade dos arcos palmares. Através da metodologia descrita, não houve complicação infecciosa ou isquêmica em nenhum dos pacientes de nossa série, o que tornaria proibitivo o enxerto.

O preparo e o manuseio adequado desses enxertos exige equipamento cirúrgico mais sofisticado e demanda maior tempo para sua execução. A experiência adquirida nas anastomoses aórticas ou coronárias com a veia safena muitas vezes é substituida por enxertos que exigem fios cirúrgicos mais caros e lupas de grande aumento. O tempo de circulação extracorpórea e o de pinçamento aórtico fatalmente se elevam no período inicial, quando o cirurgião se readapta para tais tipos de microanastomoses. Soma-se a isso a falta de estudos em larga escala a longo prazo desses novos enxertos arteriais, em detrimento do reconhecido bom funcionamento imediato dos enxertos venosos.

A AR vem sendo reconhecida como uma boa alternativa na revascularização miocárdica $(5,6,18,19)$. Seu comprimento, em média superando os $18 \mathrm{~cm}$, permite alcançar todos os ramos das coronárias. Seu diâmetro, geralmente acima de $3 \mathrm{~mm}$, possibilita a realização de várias anastomoses seqüenciais sem que haja constricção de sua luz. O extenso estudo anatômico e histológico realizado constatou a presença de uma fina camada íntima, membrana elástica interna com 4 camadas de fibras elásticas, contrastando com uma maior concentração de colágeno em sua espessa camada média, especialmente próxima à adventícia. A predominância de fibras colágenas sobre as elásticas conferem à AR uma estrutura "muscular", diretamente responsável pelos espasmos observados nos estudos preliminares de CARPENTIER et al. (7).

Segundo CALAFIORI et al. (18) a anastomose direta da AR na aorta seria uma das causas que poderia predispor ao insucesso do enxerto, por dois motivos: a diferença de espessura da parede do enxerto e a da aorta poderá gerar dificuldades técnicas na anastomose, com comprometimento do fluxo. Isso explicaria o fato de alguns enxertos livres (especialmente de $\mathrm{ATI}$ ) realizados diretamente na 
Dallan L A, Oliveira S A, Jatene F B, Corso R, Iglésias J C, Prates N, Souza J M, Verginelli G, Jatene A D - Artéria radial na ampliação do uso de enxertos arteriais para revascularização do miocárdio: consideraçōes anatômicas e tática cirúrgica. Rev Bras Cir Cardiovasc 1996; 11 (2): 75-81.

aorta terem comportamento similar aos enxertos com veia safena no primeiro ano de pós-operatório. Após esse período, eles assumiriam o mesmo resultado dos enxertos arteriais pediculados (1). Visando prevenir esse fato, no início de nossa experiência realizávamos primeiramente a anastomose da AR na aorta, o que permitia testar seu funcionamento, antes de suturá-la à artéria coronária. O segundo (e mais importante) problema decorrente da anastomose direta da AR na aorta estaria relacionado ao aumento abrupto da pressão no enxerto. Essas artérias normalmente são ramos de terceira ou quarta ordem da aorta, submetidos a regime pressórico inferior ao da aorta. Essa tensão aumentada no conduto poderia resultar em reação intimal e espasmo da parede arterial, com desenvolvimento prematuro de hiperplasia.

Temos utilizado, nos últimos meses, a anastomose do coto proximal da AR diretamente na ATle, a qual é habitualmente anastomosada ao RIA. Essa técnica, além de manter a condição hemodinâmica do enxerto, economiza em $5 \mathrm{~cm}$ ou mais o comprimento da artéria, permitindo com folga anastomoses seqüênciais com as artérias da parede lateral e inferior do ventrículo esquerdo.

Com o advento dos bloqueadores de canais de cálcio e o aprimoramento cirúrgico, a AR tem se mostrado um enxerto arterial alternativo na revascularização do miocárdio. Em muitos casos pudemos revascularizar a coronária direita, 2 ou mais ramos marginais da artéria circunflexa, a artéria diagonal e o RIA, utilizando apenas uma AR e a ATle. Acreditamos que as anastomoses seqüênciais aumentam o fluxo e mantêm pérvia a AR. As artérias AGEd e ATId in situ ou como enxerto livre têm complementado a procedimento, visando ao emprego máximo de enxertos arteriais na revascularização do miocárdio.

Dallan L A, Oliveira S A, Jatene F B, Corso R, Iglésias J C, Prates N, Souza J M, Verginelli G, Jatene A D . Radial artery for a wider arterial myocardial revascularization: microscopical anatomy and surgical technique. Rev Bras Cir Cardiovasc 1996; 11 (2): 75-81.

ABSTRACT: The aim of our study is to determine the microscopical anatomy imunohistochemistry and electronical scanning analysis of the radial artery (RA); 269 patients underwent myocardial revascularization with a RA graft were studied, performing 319 RA anatomoses; $80.7 \%$ patients were male and $93.7 \%$ caucasians. The left internal thoracic artery (LITA) was used in 246 patients at the present surgery and 17 LITA were used before (redo-operation), performing $97.8 \%$. LITA conduits employed altogether. The patients received another additional conduits: $59(21.9 \%)$ right internal thoracic arteries (RITA); $17(6.3 \%)$ right gastroepiploic arteries (RGEA); $161(59.8 \%$ ) saphenous vein grafts. An average of 3.4 anastomoses/patient were constructed. There was no post-operative complications such as ischemia or infection envolving the hand with interruption of the RA supply. The RA immunohistochemistry and electronical scanning microscopy showed that the internal elastic layer is developed and the media layer presented muscular fibers sheafs surrounded by collagen fibers more than elastic fibers. The post-operative complications include respiratory distress $(21-7.8 \%)$, atrial fibrillation (21-7.8\%), and enzymatic or electrocardiographic signs $(12-4.5 \%)$ of acute myocardial infarction. Six $(2.2 \%)$ patients needed mechanical support with intraortic balloon pump. Early angiographic controls have been performed in 21 patients. One patient developed RA dissection during the angiographic study; in all other patients the RA patency was $100 \%$. The overall in-hospital mortality rate was $2.2 \%$ (6 patients). Recently, different arrangements of the arterial grafts were adopted to achieve a more complete myocardial revascularization. The RA are frequently employed to sequential anastomosis with the inferior and lateral left ventricular coronaries. The RA proximal anastomosis is often performed with the LITA. Both the RGEA and the RITA are used as complementar arterial grafts. From 64 patients submitted to myocardial revascularization with RA last 3 months, 2 arterial conduits were performed in $62(96.9 \%)$ patients, 3 arterial conduits were performed in $27(42.2 \%)$ patients and 4 or 5 arterial conduits were performed in 8 $(12.5 \%)$. Although the pedicled internal thoracic artery graft remains the primary arterial conduit for myocardial revascularization, the radial artery is an excellent additional bypass conduit.

DESCRIPTORS: Radial artery, transplantation. Myocardial revascularization, methods. Thoracic arteries, surgery. Aorta, surgery. Saphenous vein, surgery. 
Dallan L A, Oliveira S A, Jatene F B, Corso R, Iglésias J C, Prates N, Souza J M, Verginelli G, Jatene A D - Artéria radial na ampliação do uso de enxertos arteriais para revascularização do miocárdio: considerações anatômicas e tática cirúrgica. Rev Bras Cir Cardiovasc 1996; 11 (2): 75-81.

\section{REFERÊNCIAS BIBLIÓGRAFICAS}

1 Barner H B, Naunheim K S, Fiore A C, Fischer V W Harris $\mathrm{H} \mathrm{H}$ - Use of the inferior epigastric artery as a free graft for myocardial revascularization. Ann Thorac Surg 1991; 52: 429-37.

2 Barner H B, Standeven J W, Reese J. Twelve-year experience with internal mammary artery for coronary artery bypass. J Thorac Cardiovasc Surg 1985; 90: 668-75.

3 Zeff $\mathrm{R} \mathrm{H}$, Kongatahworn $\mathrm{C}$, lannone L A - Internal mammary artery versus saphenous vein graft to the left anterior descending coronary artery: prospective randomized study with 10 year follow-up. Ann Thorac Surg 1988; 45: 451-4.

4 Sauvage L R, Wu H, Kowalsky T E et al - Healing basis and surgical techniques for complete revascularization of the left ventricle using only the internal mammary arteries. Ann Thorac Surg 1986; 42: $449-65$.

5 Acar C, Jebara V A, Portoghese M et al. - Revival of the radial artery for coronary artery bypass grafting. Ann Thorac Surg 1992; 54: 652-60.

6 Calafiori A M, Di Giammarco G, Luciani N, Maddestra N, Di Nardo E, Angelini R - Composite arterial conduits for a wider arterial myocardial revascularization. Ann Thorac Surg 1994; 58: 185-90.

7 Carpentier A, Guermonprez J L, Deloche A - The aortato-coronary radial artery bypass grafting: a technique avoiding pathologycal changes in grafts. Ann Thorac Surg 1973; 16: 111-21.

Chardigny C, Jebara V P A, Acar C A et al - Vasoreactivity of the radial artery: compartson with the internal mammary and gastroepiploic arteries with implications for coronary artery surgery. Circulation 1993; 88: (suppl.2): 115-27.

9 Curtis $\mathrm{J} \mathrm{J}$, Stoney W S, Alford Jr W C, Burrus G R, Thomas Jr C S - Intimal hyperplasia: a cause of radial artery aorto-coronary bypass graft failure. Ann Thorac Surg 1995; 20: 628-35.
10 Puig L B, Ciongoli W, Cividanes G L - Inferior epigastric artery as a free graft for myocardial revascularization. J Thorac Cardiovas Surg 1990; 99: 251-55.

11 Reyes A T, Frame R, Brodman R F - Technique for harvesting the radial artery as a coronary artery bypass graft. Ann Thorac Surg 1995; 59: 118-26.

12 Suma $H$, Wanibuhi $Y$, Terada $Y$, Fukuda $S$, Takayama $T$, Furuta $S$ - The right gastroepiploic artery graft: clinical and angiographic midterm results in 200 patients. J Thorac Cardiovasc Surg 1993; 105: 61523.

13 Van Son J A M, Smedts F, Vincent J G, Van Lier H I $\mathrm{J}$, Kubat K - Comparative anatomic studies of various arterial conduits for myocardial revascularization. $J$ Thorac Cardiovasc Surg 1990; 99: 703-7.

14 Loop F D, Lyttle B W, Cosgrove D M - Influence of the internal mammary artery graft on 10-year survival and other cardiac events. N Engl J Med 1986; 314: $1-6$.

15 Fiori A C, Naunheim K S, Dean P - Results of internal thoracic artery grafiting over fifteen years: single versus double grafts. Ann Thorac Surg 1990; 49: 202-9.

16 Galbut D L, Traad E A, Dorman M J - Seventeen-year experience with bilateral internal mammary artery grafts. Ann Thorac Surg 1990; 49: 195-201.

17 Buche M, Schoevaerdts J C, Lavagie Y - Use of the inferior epigastric artery for coronary bypass. J Thorac Cardiovasc Surg 1992; 103: 665-70.

18 Calafiori A M, Teodori G, Giammarco G, et al. - Coronary revascularization with the radial artery: new interest for an old conduit. J Card Surg 1995; 10: 140-6.

19 Dallan L A, Oliveira S A, Corso R C, et al. Revascularização do miocárdio com a artéria radial. Rev Bras Cir Cardiovasc 1995; 10:77-83. 